\title{
Monitoring and Evaluating Disaster Risk Management Capacity
}

This practice-oriented article focuses on improving the monitoring and evaluation of DRM capacity development initiatives. The paper first explores the complexities and challenges presented in the literature, before using empirical data from a research project in six countries (Ethiopia, Pakistan, Myanmar, Philippines, Haiti and Mozambique) to discuss current approaches to M\&E of DRM capacity strengthening interventions. This is generally an area of technical weakness in the initiatives studied, with poor understanding of terminology, little attention to outcomes or impact and few independent evaluations. The need for greater inclusion of participants in M\&E processes is identified and one programme from the fieldwork in Mozambique is presented as a case study

example. The article ends by presenting a unique M\&E framework developed for use by DRM programmes to track the outcomes of their interventions and ultimately raise standards in this area.

\section{Introduction}

The importance of strengthening capacities for disaster risk management (DRM) has been a growing theme of international intervention (UNDP 2008, CADRI 2011). It is important for the international community to better understand the outcomes and impact of work in this area, particularly in low-income countries that typically face the highest disaster risks and yet are the most resource constrained (Hagelsteen and Becker 2013). However, academic and non-academic literature has noted a weakness in relation to M\&E of both DRM and climate change adaptation (Bours et al. 2015, Benson and Twigg 2007, Villanueva 2011), which threatens to undermine future performance and international best practice. This practice-oriented paper provides an overview of current approaches to monitoring and evaluating DRM capacity development in low and middleincome countries, and presents a framework that could be used by programmes to track key DRM outcomes.

\section{Methodology}

This paper results from wider research examining multiple aspects of DRM capacity development.

Research began with a literature review, exploring academic and non-academic documents on M\&E in relation to DRM capacity development. DRM capacity development was defined as the process by which people, organisations and societies strengthen and sustain their abilities to take effective decisions and actions to reduce disaster risk (Few et al, in press). Few documents were identified, and so resources that considered M\&E of capacity development generally, and M\&E for climate change adaptation and DRM programmes, were included.

The research team undertook fieldwork in six countries: Ethiopia, Haiti, Pakistan, Philippines, Mozambique and Myanmar. These countries were carefully selected to give breadth to the research. Together the selection covers a wide variety of contexts, in terms of geography, types of

disaster, levels of DRM infrastructure and governance environment. Fragile and conflict affected states were also deliberately included to test whether there were specific findings related to such countries. The research team aimed firstly to investigate how effectively M\&E is being addressed in relation to DRM capacity strengthening interventions in low- and middle-income countries, and secondly to test, validate and refine an M\&E framework and supporting guidance notes for future use globally.

To investigate current M\&E practice in relation to DRM interventions, the team focused on two or three DRM capacity strengthening programmes in each of the case study countries. Programmes that were considered to be likely to provide positive examples of best practice were prioritised.

Staff were asked to describe and assess M\&E practices in semi-structured interviews, and share 
tools and reports. Donors, beneficiaries and partners were also interviewed. Responses were collated and analysed across countries to identify themes.

The team developed a draft M\&E framework with suggested indicators and supporting guidance.

Sections were tested in six national workshops across the case study countries with 110 DRM professionals from national governments, Red Cross/Red Crescent, national and international nongovernment organisations (NGOs) and donor agencies participating.

The framework was based on three proposed outcomes, each of which was tested in at least two case study countries. Participants were divided into groups to discussed the indicators, and the supporting draft guidance notes. Research team members facilitated the group exercises to ensure accurate understanding of the exercise and to provide translation support where necessary. Documentation was provided in English and local languages where necessary. Two further group semi-structured interviews were held in Pakistan and Mozambique with M\&E professionals to gain more in-depth perspectives. Responses from the workshops were collated and analysed, and revisions were made to the overall framework and guidance notes after each case study.

\title{
Observations from the literature
}

There is a wide literature focused on capacity development in low-income countries drawn from

governance, public management, development studies and organisational development disciplines. Unfortunately, this literature has tended to note shortcomings in approaches, inadequacy of tools and entrenched barriers to reform that have meant that progress on improving capacities has generally been slow and disappointing (World Bank 2005, Baser 2011b, Matheson 2009, Keijzer 2013). A key theme in the literature is that capacity development initiatives are most effective when there is high local ownership, and when programmes have been tailored to the local political and socio-economic context, building on existing capacities and needs clearly identified by prior capacity assessments (Hagelsteen and Becker 2013, Christoplos et al 2014, OECD 2006). Capacity development is no longer regarded as a purely technical process of skills-transfer, but

authors recognise the importance of the political context and governance environment as enabling, or blocking, progress. Capacity development is therefore not just about improving knowledge and skills or individuals and organisations, but is also about improving the enabling environment and the quality of institutions (Christoplos et al 2014, Lucas 2013).

\begin{abstract}
Although less research has been done on DRM capacity development, these observations are stil relevant, and authors also note problems with sustainability, lack of attention to pre-existing capacity, vague terminology, an over-reliance on training and difficulty in measuring changes in capacity (Hagelsteen and Becker 2013). Even less attention has been paid specifically to M\&E for DRM capacity development interventions, and limited published resources are available. It is also acknowledged that across the whole DRR field there are few resources such as tailored methods and tools specifically related to M\&E of disaster risk reduction (Villanueva 2011; Benson and Twigg 2007). As a result there is no common methodology that is widely used for monitoring or evaluating DRM capacity strengthening interventions, although there are some collections of DRM indicators and evaluations. The literature on resilience has produced indicators, or lists of characteristics, of disaster resilience at the national and community levels (Schipper and Langston 2015, Twigg 2009, IFRC 2011) and capacity indicators are used in various Vulnerability and Capacity Assessments (ACF 2012, IFRC 2006). However, it is important to note that these tend to measure capacity levels, rather than the effectiveness of 'capacity development' interventions. Strides have

also been made with improved tools and resources on M\&E for climate change adaptation in recent years (see for example, Adler et al. 2015 and Krause et al 2015) despite a lack of previous academic reflection on the topic (Bours et al. 2015).
\end{abstract}


The increased application of results-based management principles to development interventions in general has led to a greater focus on M\&E across all sectors. However, measuring results of capacity strengthening interventions in any field has emerged as an area of particular difficulty.

Several authors argue that using a results approach to measure capacity development interventions is problematic as it typically fails to capture 'softer' elements of capacity strengthening, and can lack flexibility (Lucas 2013). Such approaches are often more suited to capturing discrete, visible or easily quantifiable results from short-term programmes, assuming a linear progression. In contrast the development of capacity is rarely easily observable over the short term and evades easy measurement (Baser 2011a and Baser 2011b).

If there are practical and conceptual problems with M\&E of capacity development in general, these are only exacerbated when DRM, or climate change adaptation, is the focus of the activities. For example, climate change is characterised by long time frames, where the situation and context is always changing and one is essentially tracking a moving target (Bours et al 2015). Evaluation in relation to DRM also potentially means measuring non-events, and the subject matter spans multiple sectors, scales and interventions. However, universal indicators are difficult to develop as DRM and climate change adaptation always need to be grounded in the local context, scale, sector and nature of the endeavour (Chong et al 2015, Bours et al. 2015).

\section{Current approaches to M\&E of DRM capacity strengthening}

Given the problems outlined in the literature, it is perhaps unsurprising that findings from the fieldwork confirm that M\&E is indeed a problem area for most DRM capacity strengthening programmes, and progress remains very slow in developing effective frameworks that are rigorously applied in low and middle-income countries. For most of the programmes studied indepth, strong M\&E systems were not in place. Where monitoring was done it tended to focus on internal monthly updates, or lessons-learned exercises, rather than rigorous tracking of progress against pre-determined indicators at strategic points in a programme.

Programme staff were often insufficiently trained in M\&E terminology or processes, and often required support from headquarters to bolster performance in this area. A consistent theme that

emerged across the M\&E workshops in the case study countries was a confusion over various M\&E concepts and terminology. Participants (all DRM policy-makers or programme implementers) frequently requested 'step by step' instructions, simplified guidance and worked examples to help them understand the materials being presented. Terminology was frequently criticised for being 'abstract' or 'complex'. This was not necessarily just reserved for M\&E terms, words such as 'incentives', 'vulnerable' and 'resilience' produced confusion, and there was a tendency to view 'capacity building' as meaning just training or awareness raising rather than incorporating elements of institutional or organisational development.

The fieldwork also confirmed that programmes are typically focusing on activities and outputs,

rather than outcomes and impact. Of the 13 programmes studied in-depth, only one had an $M \& E$ system that focused on monitoring outcomes. Three programmes had M\&E systems that focused on outcomes and outputs and the remaining nine programmes focused just on monitoring activities and outputs. This means that most DRM capacity development programmes cannot provide robust evidence of their outcomes or ultimate impact. For example, programmes are typically using indicators such as 'number of participants at a workshop', but they are not subsequently measuring the extent to which the individuals have been able to utilise the training, or the wider organisational impact. This is not surprising, as outputs and activities are easier to monitor than outcomes and impact, which can only be determined after several years. 
A similarly concerning finding emerging from the fieldwork is that programmes appear to be rarely evaluated. Out of the 13 initiatives studied in-depth, only four conducted internal evaluations or end of project assessments, and none had external independent evaluations. Given that

programme selection was biased towards programmes that were perceived likely to be 'good performers' (following review of project documents and discussion with locally based partners), it is possible that this standard would be even lower across all DRM capacity development interventions.

It is therefore possible to conclude from the fieldwork findings that M\&E for DRM capacity strengthening interventions is generally weak and, as a result, the international community cannot provide robust evidence of the outcomes, or ultimately of the impact, of these types of programmes on reducing disaster risk.

\section{Developing sustainable and participatory M\&E systems}

In the field research it became apparent that M\&E is viewed mainly as an obligation to the donor rather than an opportunity to improve programme effectiveness. Some interviewees explicitly noted that M\&E activities were undertaken only if required by the donor. This represents a missed opportunity for programmes - Simister and Smith (2012) note that viewing M\&E as a priority only because of donor accountability risks undermining internal learning opportunities. However, it does mean that there is at least some incentive for change - programmes are typically keen to ensure good relations with their funders and so have to adhere to M\&E reporting requirements when they are in place.

A preferred approach would be a 'locally owned', more sustainable, M\&E system, that feeds back useful performance information to programme managers, but also, crucially, involves those who have been identified as 'targets' for the capacity strengthening activities. At the local government and community levels in Pakistan and the Philippines the research findings demonstrated that DRM stakeholders were more inclined to act on what they had learned if they took an active role in

using the monitoring process to identify their own capacity gaps, and subsequently identifying their own solutions to closing those gaps. Similar findings emerged from the recent Pilot Programme for Climate Resilience (PPCR) programme which recognised that local participation improved ownership in the programme using M\&E (Roehrer et al 2015). Participation is particularly important in a DRM context, because the best knowledge of both vulnerability and hazards typically lies with the communities themselves (Hagelsteen \& Becker 2013). Involving target groups in the M\&E process can therefore provide an opportunity to improve programme effectiveness, enhance ownership of the capacities that are built and therefore ultimately improve longer-term sustainability.

\section{Monitoring inclusion}

Linked to the issue of ensuring participation in M\&E processes is a need to ensure M\&E data incorporates different social groups and can be disaggregated, for example by gender. It is widely accepted that disaster risk is fundamentally gendered (Enarson et al. 2007) and that gender dimensions also need to be taken into account in disaster response and recovery (Dung et al. 2012, Harvey et al. 2012). It is therefore important to integrate gender into M\&E systems for DRM capacity strengthening initiatives. From our field research, it emerged that programme and project managers were aware of the importance of gender, but this did not always extend to incorporating indicators related to gender into their M\&E systems or disaggregating monitoring data by gender. In some cases, attention to gender considerations was completely absent from the planning process. 
In all the case study countries, the idea of monitoring gender issues was reduced to ensuring female participation in capacity development activities. For example, a programme in Myanmar had been actively monitoring and promoting "gender balance" in DRM community structures

through increased female participation in DRR committees. Although it is important to collect monitoring data on these types of activities, there is a need to move towards a more sophisticated measurement of how a programme contributes to issues of power relations between men and women, differential access to and control over resources, and leadership in decision-making processes.

The research team did not find evidence that the programmes selected had typically considered, or monitored, inclusion of other vulnerable and marginalised groups as part of their DRM capacity development activities. 'Vulnerability' tended to be considered in relation to geographical areas at risk from hazards rather than in relation to the variation in risk for different social groups within those areas.

\section{A case study example}

Although the field research identified a major deficiency in M\&E skills and processes in relation to DRM capacity development, one programme provided a useful case study example on how improved M\&E practice can be implemented in a low-income country context. In Mozambique, the

Institutionalising Disaster Prevention in Mozambique Programme (PRO-GRC) provided an improved example of M\&E systems and operations. The programme was conducted by the German Development Cooperation (GIZ) in partnership with the National Institute for Disaster Management (INGC). It was notable that this was the only case study intervention where all programme respondents spoke favourably of the M\&E system and its ability to improve performance in the short and longer-term. This success is likely to have been facilitated by the good relations between the bilateral donor and the recipient government, built up over a long period, the strong political will for DRM and robust DRM infrastructure in the country and the relatively high levels of functional institutions in relation to many other African countries. Whilst the same success might not be possible in contexts without this foundation, from interviews, several elements related to M\&E system design appear to have contributed to the effectiveness of the M\&E system, and may be transferable to other situations.

Firstly, adequate training was provided to the team at the beginning of the programme. This was a week-long training course on how to understand and use the M\&E system. Interviewees argued that it contributed to more effective programme planning and collaborative working. Secondly, PRO-GRC was the only programme studied where the M\&E system was focused on outcome indicators. These were agreed together by the governments of Mozambique and Germany. Once outcome indicators had been agreed, the managers had maximum freedom to adapt activities and outputs so they were appropriate to the project and the local situation, but would ultimately contribute to the pre-determined outcomes. This level of flexibility was highly appreciated and valued by both GIZ and INGC. Thirdly, the M\&E system was deliberately participatory, and offered opportunities for open and solution-focused discussions with partners. INGC were deeply involved in the programme and this ownership helped to institutionalise M\&E, thereby improving the sustainability of the system and the capacities developed in this area. The M\&E systems put in place by PRO GRC were still actively being used at the time of the research visit, three years after the programmeended. 


\section{A generic outcome-based M\&E framework for DRM capacity strengthening}

The research findings show that across the case study countries the quality and robustness of programme monitoring and evaluation can be substantially improved. The review of the literature above shows that there have been major problems with appropriate design and implementation of capacity development programmes in recent years. It is not expected that improving just the M\&E elements of a programme can redeem a capacity development initiative, if it has been badly conceived. However, M\&E systems can support ownership by improving participation, and can provide useful feedback loops for programme management (Simister and Smith 2012) and for the

donor community to help to track progress in tackling some of the major issues of concern in relation to capacity development. For example, this next section of the paper presents an overarching M\&E framework for DRM capacity strengthening that deliberately tries to focus the programme's attention on monitoring and evaluating not only skills transfer but the sustainability of capacities developed. It also aims to assess how well attention is actually being oriented away from technical training programmes towards improving the institutional environment and building the political will for DRM.

It is very challenging to create a universally applicable M\&E framework for DRM capacity development, because of the breadth of activities, scales and contexts in which the framework could be used. It was therefore decided to develop a flexible, outcome-focused framework, which could be tailored to the particular type of DRM activities being undertaken.

The framework presented below has been theoretically tested in each of the case study countries, but would benefit from further refinement following practical implementation by DRM organisations. This would likely require a level of financial resources to support training and guidance, as well as for the conduct of surveys and interviews. Programmes would therefore need to budget additional funds and staff time in order to use this approach. There may well be resistance from existing M\&E staff, as the approach suggested here is more challenging and resource intensive than monitoring lists of activities. There may well also be resistance from programme management nervous about how well their programme may be judged if attention switches from monitoring activities and outputs to longer-term outcomes.

The intention is that the framework presented below should supplement the local project M\&E system. Each individual project would need to generate its own activity and output indicators that would be very project specific, with related information coming from project administrative data.

Monitoring and evaluating outcomes, however, is more challenging and will often require either specific surveys or linking outcomes to more aggregate indicators collected at district or national level.

Three overarching outcomes are proposed, each with two accompanying sub-outcomes. Any DRM capacity intervention should contribute to at least one of these outcomes or sub-outcomes, and

develop appropriate specific indicators to assess progress.

Insert Table 1: M\&E Framework

\section{Measuring retained knowledge and behaviour change}

The first proposed outcome for DRM capacity development is that the ability of actors to use knowledge, innovation, education, communication and/or technology for DRM has been enhanced'. The majority of capacity development interventions will be able to adopt this outcome. The emphasis is very deliberately on the use of knowledge, equipment and skills, rather than just 
acquisition. It is therefore not simply about measuring the number of participants in training (although this would be a valid output indicator) but focuses on how much have they been able to use their improved capacities to improve DRM. Ideally a programme would measure retained learning and, where possible, whether behaviour has changed as a result of the capacity development activity. A possible indicator could be the percentage of individuals who have participated in a capacity development activity and are now using their enhanced skills, for example in relation to DRM planning and budgeting.

This outcome has two associated sub-outcomes: either individuals and communities at risk of disaster are able to use enhanced DRM skills and knowledge as a result of the capacity development programme, or actors engaged in policy making, planning and/ or implementation of DRM at national, regional, district and/or community level are using enhanced skills built by the capacity development programme. These sub-outcomes acknowledge that individuals may be engaging in activities with a view to improving their own capacities to reduce their disaster risk, or to contribute to reducing the risk of others through improved organisational DRM, at all levels.

Data can be collected either through a survey (for activities aimed at individuals) or a focus group discussion (for activities aimed at communities), ideally at baseline and again at least one year after the activity. The data should be disaggregated by gender and by vulnerable group, as appropriate.

\section{Measuring improvements in the DRM institutional framework}

The second potential outcome for DRM capacity development interventions is that 'the institutional framework for DRM has been strengthened'. During fieldwork it was observed across all case study countries that capacity development is often perceived as being the provision of equipment or training. Attention to more functional aspects of DRM is often missing, but is very important if capacity development is to be sustainable (Hagelsteen and Becker 2013, Matheson 2011, UNDP 2008, CADRI 2011). This outcome has therefore been developed to specifically focus on the importance of building functional capacity, which can be described as seeking to strengthen the organisational context for DRM, for example through improved planning, decision-making, project management and policy-making.

An 'institutional framework' refers to the systems of laws, regulations, procedures, conventions, customs and norms that shape societal behaviour. Research participants providing feedback often assumed that this outcome was only relevant at the national level, and could not be applicable at community level. On the contrary, an 'institutional framework' exists at all levels of governance,

and could incorporate informal governance arrangements as well as more formal, documented systems. The proposed outcome can therefore apply equally to village level DRM decision-making procedures, committees and plans, as to the sub-national and national levels.

The sub-outcomes focus on tracking progress on, and wide inclusion of stakeholders in, developing policies, strategies and procedures. Possible indicators could be, for example, the submission of an Act to parliament, revision of local planning procedures following consultation with at-risk groups, or joint-working between ministries leading to changes in budgeting procedures. Given how long such change processes can take, it is important to consider an appropriate timeline and interim indicators may be necessary. Establishing the baseline situation will be important. Someone working on the programme should be tasked with documenting and tracking the status of policies, strategies and procedures at the start of activities and monitor any changes for the duration of the programme. In terms of monitoring participation, the programme should set up a tracking system which covers the various forms of consultation (open meetings, econsultations, round tables) and communications linked to the development of policies and strategies. A target should be set in terms of inclusion of particular groups of the population, but a quantitative target may not be appropriate, and qualitative process indicators may also be useful. 


\section{Measuring the creation of an enabling environment}

The importance of creating the motivation to prioritise DRR in society is increasingly emphasised in the literature (CADRI 2011, WDR 2015). The third potential outcome in the framework therefore focuses specifically on whether an intervention has been able to build an enabling environment and 'motivation to achieve effective DRM has been improved'.

The suggested sub-outcomes show that an enabling environment can be built either through the creation of political support for DRM or through improving the motivation of individuals and communities to reduce their own disaster vulnerability. Potential indicators in this area are likely to

measure action taken as a result of awareness-raising, for example politicians using DRM information in a speech or draft legislation, changes to community activity following the lobbying of traditional leaders, or the percentage of the population showing changed behaviour following a media campaign. Most capacity building programmes can incorporate some activities aimed towards building an enabling environment for DRM, and so this outcome can still be incorporated into a programme's M\&E system when awareness-raising and lobbying is not the main focus of activity.

Indicators for this sub-outcome are likely to measure whether an action has been taken as a result of awareness-raising activities. It is important that programmes consider how activities will realistically lead to raising awareness or political support for DRM, and at what level. Ideally programmes will develop a 'theory of change' explaining how programme activities and outputs will link to this outcome. If the activity is direct lobbying of policy-makers and decision-makers, it may be possible to identify results in terms of specific actions, but it is more likely that measurement of the indicator will involve interviews and surveys. If the capacity development activity is aimed at a general audience, then a survey (preferably longitidunal) will be useful to track behaviour change.

\section{Conclusion}

The research highlights that DRM capacity development programmes typically need help to develop and implement robust M\&E systems and to shift their focus from activities and outputs, to outcomes and impact. Fieldwork also showed that when incentivised by donors and funding partners requiring certain M\&E reports and practices, DRM programme implementers were willing to improve their M\&E systems and activities. The framework presented shows how improvements to M\&E systems, whilst not a silver bullet to resolve all the barriers to bringing sustainable institutional development in relation to DRM, could potentially orient programme implementers to tracking more meaningful data around the strengthening of functional, institutional capacity and the generation of an enabling political environment. Access to this information at least gives programmes and donors a better idea of their effectiveness and can focus attention on institutional barriers to, and opportunities for, the development of sustainable DRM capacity. 


\section{References}

ACF (2012) 'Participatory Risk, Capacity and Vulnerability Analysis: A Practitioner Manual for Fieldworkers', Action Contre le Faim.

Adler, R., Wilson, K., Abbot, P., \& Blackshaw, U. (2015) 'The Case of the United Kingdom's Investment to Ethiopia's Climate-resilient Green Economy' New Directions for Evaluation, issue 147, pp.61-74.

Baser, H. (2011a) 'Managing for Capacity Results', Cairo Workshop on Capacity Development, OECD.

Baser, H. (2011b) 'Perspectives on Capacity Development in Fragile Situations', Learning Network on Capacity Development.

Benson, C. and Twigg, J. (2007) ‘Evaluating Disaster Risk Reduction Initiatives’, Tools for Mainstreaming Disaster Risk Reduction, ProVention Consortium.

Bours, D., McGinn, C., Pringle, P. (2015) Monitoring \& Evaluation of Climate Change Adaptation: A Review of the Landscape', New Directions for Evaluation, Issue 147, pp.1-12.

CADRI (nd) ‘Basics of Capacity Development for Disaster Risk Reduction’, UNISDR.

Chong, J., Gero, A. and Treichel, P. (2015) 'What Indicates Improved Resilience to Climate Change?' New Directions for Evaluation, issue 147, pp. 105-116.

Christoplos, I., Engstrand, K. and Liljelund Hedqvist, A. (2014) 'Capacity Development Literature Review', Sida, Sweden.

Dung, L.T.M, et al. (2012) Flood Preparedness in Vietnam: A Systematic Gender-Aware Approach. Oxfam GB.

Enarson, E., Fothergill, A., Peek, L. (2007) ‘Gender and Disaster: Foundations and Directions' In: Handbook of Disaster Research. Springer, New York, pp130-146

Few, R., Scott, Z., Wooster, K., Tarazona, M and Flores Avila, M., (in press) 'Strengthening Capacities for Disaster Risk Management II: Lessons for Effective Support', International Journal for Disaster Risk Reduction, Elsevier.

Hagelsteen, M. and Becker, P. (2013) 'Challenging Disparities in Capacity Development for Disaster Risk Reduction', International Journal of Disaster Risk Reduction. 3 (2013) 4-13.

Harvey, C. et al .(2012) Post-Earthquake Response and Reconstruction. Gender-Sensitive Advocacy in Indonesia. Oxfam GB.

IFRC (2006) 'What is VCA? An Introduction to Vulnerability and Capacity Assessment', International Federation for Red Cross/ Red Crescent Societies, Geneva.

IFRC (2011) 'Characteristics of a Safe and Resilient Community: Community Based Disaster Risk Reduction Study', Arup International, International Federation for Red Cross/ Red Crescent Societies, Geneva. 
Keijzer, N. (2013) 'Unfinished Agenda or Overtaken by Events? Applying Aid- and Development Effectiveness Principles to Capacity Development Support', German Development Institute (Discussion paper 17), Germany

Krause, D., Sschwab, M. and Birkmann, J. (2015) 'An Actor-Oriented and Context Specific

Framework for Evaluating Climate Change Adaptation', New Directions for Evaluation, issue 147, pp.37-48.

Lucas, B. (2013) Current Thinking on Capacity Development. Birmingham, UK: Governance and Social Development Resource Centre, University of Birmingham.

Matheson, A. (2009) 'Formulation of a Capacity Development Framework: A Literature Review', Report to UNICEF India, Oxford: Oxford Policy Management.

OECD (2006) 'The Challenge of Capacity Development - Working Towards Good Practice', OECD, Paris.

Roehrer, C. and Kouadio, K.E (2015) 'Monitoring, Reporting and Evidence-based Learning in the Climate Investment Fund's Pilot Program for Climate Resilience', New Directions for Evaluation, issue 147, pp.129-145.

Schipper, E.L.F. and Langston, L. (2015) 'A Comparative Overview of Resilience Measurement Frameworks: analysing indicators and approaches', Overseas Development Institute, London.

Twigg, J. (2009) 'Characteristics of a Disaster-Resilient Community: A Guidance Note', UCL Hazard Research Centre, London.

Villanueva, P.S., (2011) 'Learning to ADAPT: Monitoring and Evaluation Approaches in Climate Change Adaptation and Disaster Risk Reduction - Challenges, Gaps and Ways Forward', Discussion Paper 9, Strengthening Climate Resilience (SCR).

World Bank (2005) 'Capacity Building in Africa: An OECD Evaluation of World Bank Support', World Bank Operations Evaluations Department, Washington D.C: 
Table 1: M\&EFramework

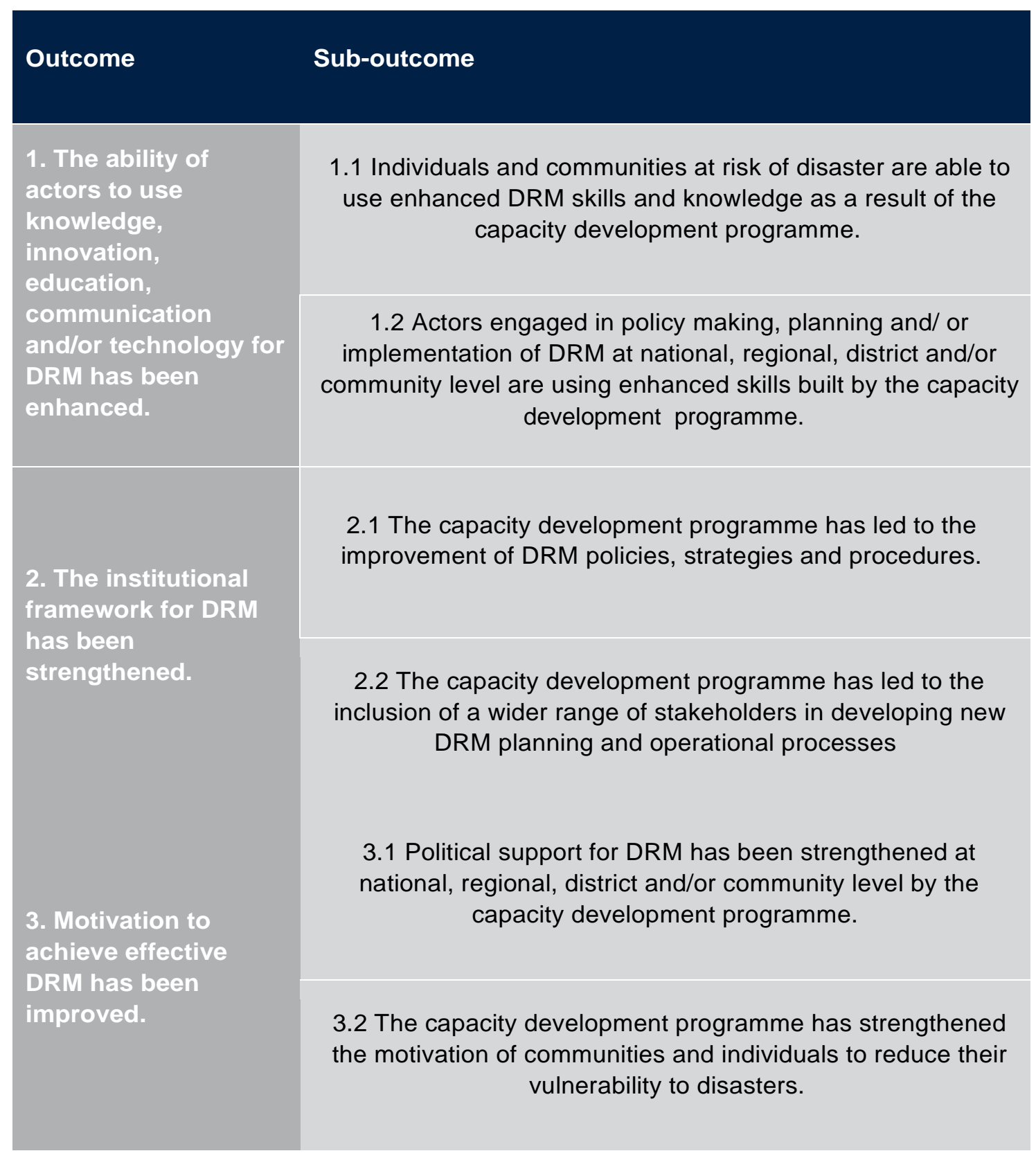

